\title{
CLINICAL PATHOLOGY IN GENERAL PRACTICE LIVER-FUNCTION TESTS
}

\author{
BY \\ N. F. MACLAGAN, M.D., D.Sc., F.R.C.P., F.R.I.C. \\ Professor of Chemical Pathology in the University of London at Westminster Medical School; Chemical Pathologist, \\ Westminster Hospital
}

In view of the very large number of liver-function tests which have been elaborated, the present discussion is limited to a few tests of established value. These tests can be divided into two groups according to the nature of the material required. Tests in the first group can be performed on material collected by the practitioner, whereas those in the second group require the attendance of the patient at a laboratory or hospital centre or, alternatively, a personal visit by the pathologist. Tests performed on material submitted by the practitioner include serum bilirubin, serum alkaline phosphatase, flocculation tests, serum albumin and globulin estimations, and qualitative urine tests for bile pigments. More elaborate tests are the quantitative estimation of urine bile pigments, bromsulphthalein retention, and prothrombin response to vitamin $\mathrm{K}$.

The blood tests in the first group require the collection of approximately $10 \mathrm{ml}$. of clotted blood reasonably free from haemolysis. To achieve this it is necessary to use a dry sterilized syringe and to remove the needle before squirting the blood gently into the dry collection tube. Urine can be naturally voided and should preferably be an afternoon specimen, since the amount of urobilinogen, for example, is small in morning specimens. It is, in general, inadvisable to rely upon any one test of liver function, and a "battery" of tests is usually employed. This need not necessarily include all the tests mentioned, and some guidance on the choice of tests is given below.

The various tests are considered individually in relation to their usefulness in the diagnosis of liver diseases.

\section{Serum Bilirubin}

It is difficult to avoid discussion of technique in this test, as so many different methods have been used since the pioneer work of van den Bergh. I prefer a separate estimation of "direct" and "indirect" serum bilirubin by a photoelectric method based on that of Malloy and Evelyn. With this technique direct pigment refers to colour developed in aqueous solution after 15 minutes, while the total pigment is read in alcoholic solution at 30 minutes. The indirect pigment represents the difference between the two values. Terms such as direct, indirect, and biphasic reaction are also used, but they are difficult to define quantitatively.

The normal total serum bilirubin is from 0 to $1 \mathrm{mg}$. per $100 \mathrm{ml}$. of serum, and a zone of latent jaundice not clinically detectable exists between 1 to $3 \mathrm{mg}$. per $100 \mathrm{ml}$, patients with values over $3 \mathrm{mg}$. being usually clinically jaundiced. The demonstration of latent jaundice is an important aid to diagnosis in conditions such as infective hepatitis, hepatic cirrhosis, and the haemolytic anaemias. In jaundiced patients both the direct and the indirect bilirubin will increase, but if more than half the total is indirect the jaundice is probably haemolytic in nature. Jaundice due mainly to increase in direct pigment is usually either obstructive or toxic and infective.

The serum bilirubin estimation is an important basic procedure as it provides an accurate record of the intensity of any jaundice which may be present. It may neverthe: less give normal results in certain cases of chronic liver disease such as cirrhosis, carcinomatosis, etc.

\section{Serum Alkaline Phosphatase}

The serum alkaline phosphatase is an enzyme produced mainly in the bones by the osteoblasts but also by the liver cells. It can therefore only be used as a liver-function test in cases in which the bones can be assumed to be normal. Its level in the blood (normal 3 to $13 \mathrm{King}$-Armstrong units) is elevated in a variety of liver conditions such as obstructive jaundice, hepatitis, carcinomatosis, abscess, amyloidosis, and cirrhosis. It is, however, on the whole much higher in obstructive jaundice than in the other conditions named, and values over 35 units, for example, are particularly suggestive of obstruction as opposed to hepatitis in a jaundiced patient. In the differential diagnosis of jaundice it is particularly valuable to combine this test with one or more of the flocculation tests.

\section{Flocculation Tests}

Under this heading are included a rather large number of empirical procedures which have been used to demonstrate hepatic dysfunction, ranging from the earlier formolgel and Takata-Ara reactions to more recent tests such as the serum colloidal gold reaction and the thymol turbidity and flocculation tests. There are, however, considerable individual differences in these various tests, and the following remarks apply mainly to the thymol and gold tests.

These tests are positive, particularly in acute infective hepatitis (about 90\%) and in cirrhosis (about 50\%), and have a special tendency to be negative in obstructive jaundice. They are therefore of value in the detection of hepatitis and in the differential diagnosis of jaundice. It should always be remembered that they are not specific tests of liver damage, as they depend upon a particular type of serum protein disturbance which may also occur in various acute and chronic infections such as glandular fever, malaria, infective endocarditis, etc. The necessity of interpreting all laboratory procedures with due regard to the whole clinical picture applies particularly to these tests.

\section{Serum Albumin and Globulin Estimations}

These tests are of most value in the more chronic types of liver disease such as cirrhosis. In cirrhosis the albumin (normal 4 to $5.5 \mathrm{~g}$. per $100 \mathrm{ml}$.) tends to fall and the globulin (normal 1.5 to $3 \mathrm{~g}$. per $100 \mathrm{ml}$.) to rise. Alternatively, one may say that the albumin/globulin ratio (A/G ratio) (normally about $2: 1$ ) tends to fall in liver disease, but as changes in the two fractions are largely independent of one another it is preferable to consider the values individually. Neither change is an entirely specific indication of liver damage, but if both alterations are present hepatic disease is the most likely explanation.

These tests are of value in confirming a diagnosis of cirrhosis. From the prognostic point of view the albumin is the more important estimation, values below $2 \mathrm{~g}$. per 100 $\mathrm{ml}$. indicating a poor prognosis, and usually being associated with an obstinate oedema. High globulin values, on the other hand, are compatible with good health and do not usually produce symptoms. These estimations, based on 
salting-out procedures, are to some extent becoming replaced by electrophoretic analysis of the serum proteins. However, this procedure is not available in all laboratory centres and rules for the interpretation of results are not yet so firmly established.

\section{Qualitative Urine Tests for Bile}

The ordinary clinical tests for bile such as the foam and iodine tests are, of course, very insensitive. The following remarks refer to tests such as the Harrison-Fouchet test for bilirubin and the Ehrlich-Watson test for urobilinogen. These tests reach their maximum usefulness in patients who are not clinically jaundiced. In such cases the occurrence of bilirubinuria suggests a latent jaundice usually due to hepatitis or to biliary obstruction, and this test may be positive some days before a rise in the serum bilirubin can be demonstrated. The test is therefore particularly valuable in the pre-icteric phase of infective hepatitis and in hepatic cirrhosis.

An increase in urine urobilinogen may accompany bilirubinuria but may also occur without any latent jaundice in chronic liver damage such as hepatic cirrhosis. It is a less specific indication of liver pathology than bilirubinuria and may occur as a temporary event in febrile states. Urobilinuria may also occur in jaundiced patients but is of limited diagnostic value in this group; on the whole, increased values are suggestive of toxic and infective jaundice, but there are many exceptions to this rule.

\section{Quantitative Estimation of Urine Bile Pigments}

As noted above, the urinary excretion of urobilinogen is increased as a result of liver damage, and this increase is best demonstrated by a quantitative estimation. Urobilinogen is formed mainly by the bacterial decomposition of bilirubin in the intestinal tract. It is partially reabsorbed from there into the blood stream and is partly re-excreted by the liver in the bile. It follows from this that urine for this test should not be collected in the morning, when the effects of bile flow will be minimal, and also that the investigation is of greatest value in patients not clinically jaundiced. In the absence of jaundice it can be assumed that bile is reaching the intestine, whereas in the presence of jaundice this may not be the case.

Although this examination can be carried out on 24-hour urine specimens it is more convenient to collect a timed specimen of urine in the afternoon and to estimate the amount of pigment in a one- or two-hour specimen by the methods of Watson. Normal values for urobilinogen are from 0 to 1 Ehrlich unit per 2 hours or up to $3 \mathrm{mg}$. per 24 hours.

The interpretation of this estimation is very similar to that given for the qualitative urobilinogen test above, but the procedure is more accurate and reliable. The test is of particular value in non-jaundiced cases of hepatitis and cirrhosis.

\section{Bromsulphthalein Retention}

Bromsulphthalein (phenoltetrabromphthalein disodium sulphonate) is a harmless dye which is injected intravenously and is excreted mainly in the bile. The recommended dosage is $5 \mathrm{mg}$. per $\mathrm{kg}$. body weight and blood is collected exactly 45 minutes after injection. The amount of bromsulphthalein remaining in the serum is estimated. Normal subjects will show no significant bromsulphthalein retention at 45 minutes, and in pathological states the amount remaining in the circulation at this time is expressed as a percentage of the injected dose to give a figure of percentage bromsulphthalein retention.

Like the preceding test this is of little value in jaundiced subjects and is mainly used in non-jaundiced subjects and in cases of hepatitis and cirrhosis. It is a sensitive and convenient test in cases of this type, although even this test is not invariably positive in cirrhosis. It will also indicate the existence of liver dysfunction in other conditions, such as malaria, diabetes mellitus, glandular fever, etc.

\section{Prothrombin Response to Vitamin $\mathbf{K}$}

This test has been used mainly in cases of obstructive jaundice but is also of value in the differentiation between surgical and non-surgical jaundice. It is, of course, applicable only to those patients who have low prothrombin values when first seen. This may happen either because the exclusion of the bile from the intestine interferes with the absorption of vitamin $\mathbf{K}$ (obstructive jaundice) or because the liver is too severely damaged to manufacture vitamin $\mathrm{K}$ from its plasma precursors (hepatitis or cirrhosis). About a quarter of all jaundiced subjects may be expected to exhibit an initial deficiency in prothrombin and therefore become eligible for this test.

After the initial estimation vitamin $\mathbf{K}$ is administered, usually as $50-100 \mathrm{mg}$. of a water-soluble preparation, and the prothrombin is again estimated after 24 to 48 hours. A rapid return towards normal indicates good liver function and confirms the diagnosis of biliary obstruction. An absence of response indicates gross liver damage and suggests a hepatogenous origin for the jaundice. In practice the first part of this test is often omitted; a low prothrombin value after adequate vitamin-K therapy is, of course, significant even in the absence of the preliminary observation.

Quite apart from its value in diagnosis the test is of great importance if operation is contemplated, as a low plasma prothrombin value indicates a greatly increased risk of haemorrhage.

\section{Combination of Tests}

Certain tests are particularly suitable for use in combination, and this applies especially to the serum alkaline phosphatase estimation and the thymol flocculation test in relation to the differential diagnosis of jaundice. The individual variations in these two tests have already been discussed, but the accuracy of diagnosis is improved when they are undertaken simultaneously. Thus cases of jaundice with negative thymol tests and serum alkaline phosphatase values above 35 units are almost invariably obstructive in nature, whereas those with strongly positive flocculation tests and/ or with normal serum alkaline phosphatase levels are usually non-obstructive. In some $20 \%$ of cases the findings fall between these two extremes and are of no diagnostic value.

\section{Indications for Liver-function Tests}

Laboratory procedures in general can only be used to answer certain restricted types of question, and it is important to have a clear idea of exactly what the provisional diagnosis is before any help can be expected from tests of this type. Most of the indications for the tests will fall under one or more of the following heads:

In suspected primary liver disease the condition may be either acute or chronic in nature-for example, infective hepatitis or hepatic cirrhosis. Any of the tests described may assist in making the diagnosis, their relative importance varying, however, with the type of case. In preicteric hepatitis the first tests to become positive are those for urine bile pigments, particularly bilirubin. At a later stage all the pigmentary tests will be positive and usually the flocculation tests in addition. In hepatic cirrhosis, on the other hand, the pigmentary tests and many of the others may be at times completely negative and more reliance is placed upon the serum protein estimations and the bromsulphthalein retention.

The liver may be secondarily involved in a variety of diseases. In heart failure, for example, the turbidity and flocculation tests are often positive and urobilinuria may occur. In malaria and glandular fever the positive flocculation tests constitute the most frequent abnormality, while in thyrotoxicosis the disturbance is of a different type and may require the use of tests such as a galactosetolerance test and a hippuric-acid test, which are not especially valuable for general diagnostic purposes. 
The differential diagnosis of hepatic enlargement tends to be concerned mainly with the problem of distinguishing hepatic cirrhosis from carcinomatosis. The biochemical changes are usually distinctly different in the two groups, the serum protein and flocculation tests being mainly altered in cirrhosis while the raised serum alkaline phosphatase is the main change in carcinomatosis.

In the differential diagnosis of jaundice we are usually trying to distinguish jaundice due to a mechanical obstruction of the bile passages from that due to hepatitis or cirrhosis. As noted above, the thymol flocculation test and serum alkaline phosphatase used in combination give the best results in cases of this type, making the distinction satisfactorily in about $80 \%$ of such cases, the results being equivocal in the remaining $20 \%$. Some assistance can also be given in the detection of haemolytic jaundice in which the indirect serum bilirubin is more raised than the direct pigment, and the serum alkaline phosphatase tends to be normal.

The tests principally used in the prognosis of liver diseases are the serum albumin estimation and the prothrombin response to vitamin $K$. In cirrhosis values for the serum albumin below $2 \mathrm{~g}$. per $100 \mathrm{ml}$. indicate an irrecoverable liver damage with a poor prognosis. In obstructive jaundice failure of a low prothrombin level to respond to vitamin-K therapy suggests grave liver damage and a poor operative risk.

The investigation of anaemias is, of course, the province of the haematologist, but the differential serum bilirubin estimation may assist in the detection of haemolysis, in which the indirect pigment is mainly increased. This finding has been found valuable in the diagnosis not only of the various haemolytic anaemias but also in cases of pernicious anaemia.

\section{Choice of Test}

As mentioned above, it is usually desirable to combine several different tests, and the following scheme may be taken as a guide.

Tests for Routine Application.-Serum bilirubin, serum alkaline phosphatase, flocculation tests, qualitative urine tests for bilirubin and urobilinogen: this small battery of tests will often give all the information required, at all events from a diagnostic standpoint. They will establish the absence or presence of jaundice, and if jaundice is present they will provide a record of its intensity and will also give a good indication of its probable cause. In the non-jaundiced patient, on the other hand, one or more of the tests may be positive and provide enough evidence to confirm a suggested diagnosis of hepatic disease.

Further Tests.-If the above tests are negative or if their indications are in any way equivocal the remaining tests mentioned may be undertaken in the following order of preference: serum albumin and globulin estimations, quantitative estimation of urine bile pigments, bromsulphthalein retention, prothrombin response to vitamin $K$. It will usually be unnecessary to do more than one or two of these additional tests in order to support, for example, a diagnosis of liver cirrhosis, but the vitamin-K test is specifically indicated in surgical jaundice.

\section{Conclusion}

In conclusion it may be emphasized that modern liverfunction tests reach a high degree of sensitivity and will rarely fail to demonstrate any significant liver damage which may be present. This implies that the disturbances of function occurring in various non-hepatic diseases may complicate interpretation, which should always be undertaken with due regard to the whole clinical picture.

Next article on Clinical Pathology.- - " Laboratory Investigations in Suspected Industrial Poisoning," by Professor R. E. Lane.

\section{Reports of Societies}

\section{CONVULSIONS IN CHILDHOOD}

The Section of Paediatrics of the Royal Society of Medicine met under the chairmanship of Dr. C. T. Potter on March 26 to discuss "The Significance of Convulsions in Infancy and Childhood."

Dr. Peter Tizard (Paediatric Unit, St. Mary's Hospital, Paddington) opened with a general review of the aetiology of convulsions. Most textbooks divided fits into the symptomatic-those caused by fever, cerebral tumour, brain injury, etc.-and the idiopathic, which included infantile convulsions, pyknolepsy, and true epilepsy. Was this sort of division justifiable? With regard to symptomatic convulsions, we did not know the effective cause, in the Aristotelian sense, of any fit. Clearly fever, for example, in itself could not be regarded as a cause. Were it so any individual would have a fit whenever his temperature reached a certain critical height, whereas in fact that happened only with an extremely small minority of children. Similarly, a focal lesion, such as a cerebral tumour or brain injury, could not in itself be the cause of a fit ; if it were, the patient would convulse continuously and not intermittently.

Probably, in the production of any one fit there were several causative factors. This view led to the concept of a "convulsive threshold," to the idea that there was no fundamental difference between the epileptic and the nonepileptic, and that any person given an adequate stimulus would have a fit. The nature and degree of stimulus would vary widely, of course; at one end of the scale were those who would convulse only in response to a severe electric shock or an injection of insulin, while at the other end the threshold was so low that fits seemed to occur spontaneously. Between these two extremes were those who developed fits in response to such precipitating factors as fever or brain injury.

This view, that we are all potential epileptics but have varying degrees of natural resistance, provided a useful method of approach to the parent of a child who had fits. It took away much of the sting that goes with a diagnosis of epilepsy. Useful though it was, however, it represented an over-simplification of the problem. There was no evidence of hereditary predisposition in some forms of symptomatic epilepsy. In conclusion, Dr. Tizard discussed the possibility that included in what we now call idiopathic epilepsy there were several different diseases of unknown aetiology and pathology, of which the main symptom was the occurrénce of fits.

\section{The First Fit}

Dr. Christopher Ounsted (Research Assistant to the Oxford Regional Hospital Board) spoke about the "First Fit." Its significance in a young child depended on the setting in which it occurred. In severe infections multiple fits were common and were of grave prognostic significance. Prolonged fits and status epilepticus were fatal per se. Dr. Ounsted gave figures showing that a sharp reduction in the mortality of purulent meningitis, and a parallel reduction in the incidence of fits, could be achieved by giving prophylactic anticonvulsants to affected children. Established convulsions should be aborted as rapidly as possible; phenobarbitone and chloral were valueless once a fit had begun, but sodium amylobarbitone given intramuscularly in doses from 0.125 to $0.25 \mathrm{~g}$. controlled convulsions rapidly and safely.

After a single fit the regular use of anticonvulsants was not necessary, but the child should be given prophylactic phenobarbitone during any future febrile illness up to the age of 4 years. This measure seemed to be effective in preventing fits, and the mothers were comforted by having some practical steps offered to them. 